\title{
An Analysis Technique for Post-Wall Waveguides
}

\author{
T.J. Coenen*11, D.J. Bekers*, J.L. Tauritz ${ }^{\dagger}$, F.E. van Vliet* \\ *TNO Defence, Security and Safety \\ P.O. Box 96864, 2509 JG Den Haag, The Netherlands \\ $1_{\text {teis.coenenetno.nl }}$ \\ ${ }^{\dagger}$ Department of Telecommunication Engineering, University of Twente \\ P.O. Box 217, 7500 AE Enschede, The Netherlands
}

\begin{abstract}
In the last decade, post-wall waveguides have emerged as an interesting building block for (antenna) feed networks because of their potential low losses, low costs, ease of manufacturing and integrability with existing printed circuit board techniques. In this paper we present a general formulation to analyze the characteristics of post-wall waveguides with metal or dielectric posts. This formulation is based on a field expansion technique, applied to infinite arrays of posts. The modal behavior of post-wall waveguides is analyzed by considering the behavior of the field expansion coefficients as function of frequency and we compare our results with Ansoft HFSS simulations and measurements.
\end{abstract}

\section{INTRODUCTION}

The post-wall waveguide, or substrate integrated waveguide, is a rectangularly shaped waveguide laterally bound on two sides by periodic arrays of posts. At the top and bottom the guide can be either unbounded or bounded by metal plates or dielectric slabs/half spaces. The cylindrical posts can consist either of metallic or dielectric material. An impression of the post-wall waveguide is given in Fig. 1. Especially at millimeter-wave frequencies, the post-wall waveguide is a promising alternative for traditional planar transmission lines as it may offer reduced transmission losses. With properly chosen constituent elements, such as metallic or air posts, the structure can be fabricated using conventional printed circuit board technology.

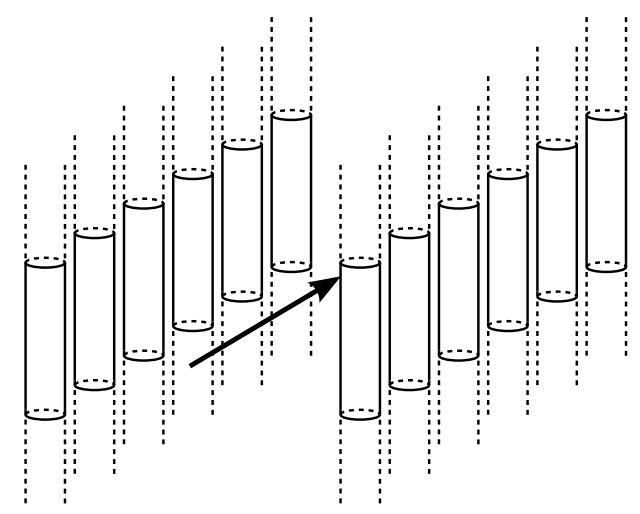

Fig. 1. Impression of a post-wall waveguide, in this case laterally bound by single linear arrays of cylindrical posts.

In the microwave literature, relevant analysis and design is, in particular, focused on waveguides with metallic posts and top and bottom plates resembling classical rectangular waveguides. Several authors address the resemblance between classical rectangular waveguides and metallic post-wall waveguides. In [1], the equivalent width is introduced to relate the characteristics of a post-wall waveguide to a rectangular waveguide. This width is computed for an infinitely long guide based on method-of-moments (MoM) code. Several approximate formulas for this equivalence have been deduced using other numerical techniques including the boundary integralresonant mode expansion (BI-RME) method [2], the finitedifference frequency-domain method and the finite-element method (FEM) [3], [4], [5].

Measurements of straight segments of metallic post-wall waveguides can be found in [6], [7]. More specifically, in [6] the loss of a post-wall waveguide is measured at $76.5 \mathrm{GHz}$. Measurements of the effective width of post-wall waveguides are treated in-depth in [8]. As an extension to transmission line modeling, initial studies on the behavior of bends and T-junctions are presented in [9] and [1], respectively. Bends, power dividers, and T-junctions are investigated in [10] for laminated post-wall waveguides.

For post-wall waveguides with dielectric posts, the available literature is more fragmented and distributed over the microwave and optical fields. In integrated optics technology the use of dielectric post-wall waveguide facilitates the realization of narrow guide widths and small curvature bends [11], [12], [13].

In the microwave field, dielectric post-wall waveguides have been introduced more recently and their full potential has yet to be explored. In [14], two walls of dielectric posts are used as a frequency selective surface, leaking power into a central guiding region. Structures with the primary goal of wave-guidance are investigated in [15], [16], [17] and [18]. In [15], a study on wave-propagation feasibility is presented based on finite-difference time-domain simulations, where the top and bottom boundaries of the guide are dielectric layers. Guides consisting of infinitely-long square posts are analyzed in [16], where the reflection at the walls is modeled by means of transmission matrices. Finally, in [17], [18] a post-wall waveguide resembling an image guide with a metal bottom plate and no top plate is designed based on FEM simulations, fabricated and tested. The same authors use the image guide to produce an antenna with a fan beam in [19]. 


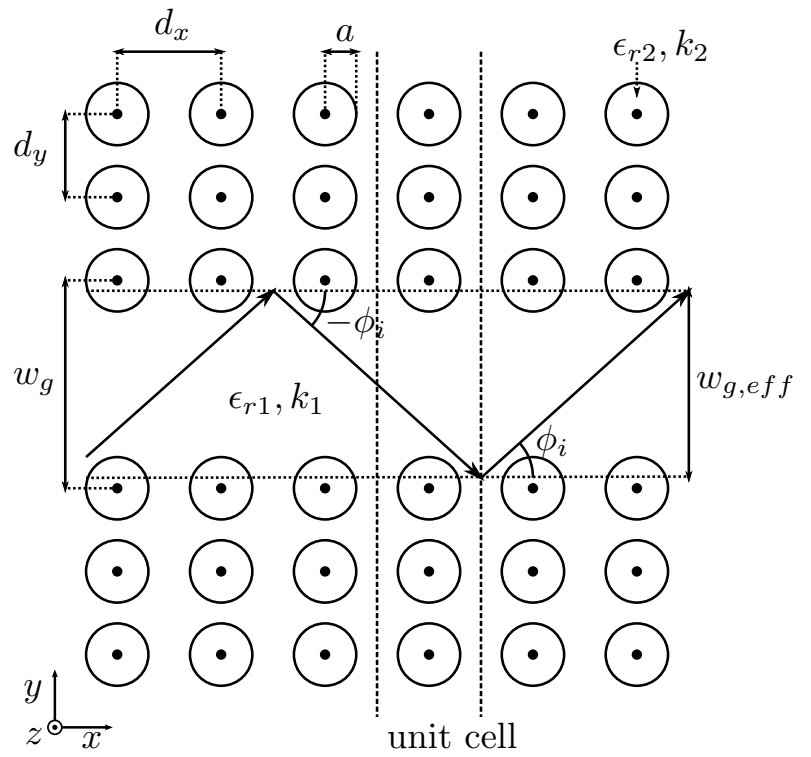

Fig. 2. Top view of a post-wall waveguide, here laterally bounded by three linear arrays of cylindrical posts.

In [20] we presented a method for analyzing finite-length post-wall waveguides based on a Galerkin MoM approach for the case of metallic posts and a field expansion method for post-wall waveguides with small dielectric posts. We took a first step toward a unified analysis of post-wall waveguides by means of a field expansion technique in [21] and we identified unwanted resonances inside the walls that can limit waveguide performance.

We extend here, the field expansion technique with a modal representation and show that this technique is suitable for characterizing post-wall waveguide components. We compare the results of propagation constant measurements of segments of straight metallic and dielectric post-wall waveguide transmission lines with theoretically obtained results.

\section{THEORY}

To clarify the notation used in this paper, Fig. 2 shows the geometry of a post-wall waveguide with three arrays of posts per side-wall.

\section{A. Field Expansion}

To simplify the post-wall waveguide modeling, we make the following assumptions

1) the posts are homogeneous,

2) the posts extend infinitely along the axial direction of the posts, and

3) only an electric field in the axial direction of the posts exists.

This reduces our task, in effect, to a $2 \mathrm{D}$ problem with the electric field solely described by the $z$-component and wave propagation can only occur in the $(x, y)$-plane. Using a similar approach as in [22], we expand the excited, scattered and internal fields of a single post in the origin of our coordinate system as Fourier series in the polar coordinates $(r, \phi)$

$$
\begin{aligned}
& E_{z}^{\mathrm{exc}}=E_{0} \sum_{m=-\infty}^{\infty} j^{-m} J_{m}\left(k_{1} r\right) e^{j m\left(\phi-\phi_{i}\right)} \\
& E_{z}^{\mathrm{sct}}=E_{0} \sum_{m=-\infty}^{\infty} A_{m} j^{-m} H_{m}^{(2)}\left(k_{1} r\right) e^{j m \phi} \\
& E_{z}^{\mathrm{int}}=E_{0} \sum_{m=-\infty}^{\infty} B_{m} j^{-m} J_{m}\left(k_{2} r\right) e^{j m \phi}
\end{aligned}
$$

where $E_{0}$ is the excitation amplitude. When we apply the condition of continuity of the tangential electric and magnetic fields on the post boundaries, we can formulate a block matrix system of equations

$$
\left[\begin{array}{cccc}
{\left[\mathbf{C}_{11}\right]} & {\left[\mathbf{C}_{12}\right]} & \cdots & {\left[\mathbf{C}_{1 \mathbf{P}}\right]} \\
{\left[\mathbf{C}_{21}\right]} & {\left[\mathbf{C}_{22}\right]} & & \\
\vdots & & \ddots & \\
{\left[\mathbf{C}_{\mathbf{P 1}}\right]} & & & {\left[\mathbf{C}_{\mathbf{P P}}\right]}
\end{array}\right]\left[\begin{array}{c}
\mathbf{A}_{1} \\
\mathbf{A}_{2} \\
\vdots \\
\mathbf{A}_{\mathbf{P}}
\end{array}\right]=\left[\begin{array}{c}
\boldsymbol{\Phi}_{1} \\
\boldsymbol{\Phi}_{2} \\
\vdots \\
\boldsymbol{\Phi}_{\mathbf{P}}
\end{array}\right]
$$

where $\Phi_{\mathbf{p}}$ represents the phase of the excitation field and $\mathbf{A}_{\mathbf{P}}$ are the unknown expansion coefficients.

The elements of $\left[\mathbf{C}_{\mathbf{p q}}\right]$, in the case of metallic posts are

$$
C_{p q, m n}= \begin{cases}0 & (p=q, \\ & m \neq n) \\ \frac{H_{m}^{(2)}\left(k_{1} a\right)}{J_{m}\left(k_{1} a\right)} & (p=q, \\ H_{m-n}^{(2)}\left(k_{1} r_{p q}\right) e^{-j(m-n) \phi_{p q}} & (p \neq q) .\end{cases}
$$

And similarly, for the case of dielectric posts, the elements are

$$
\begin{aligned}
& C_{p q, m n}= \\
& \left\{\begin{array}{lc}
0 & (p=q, \\
& m \neq n) \\
\frac{k_{2} H_{m}^{(2)}\left(k_{1} a\right) J_{m}^{\prime}\left(k_{2} a\right)-k_{1} H_{m}^{\prime(2)}\left(k_{1} a\right) J_{m}\left(k_{2} a\right)}{k_{2} J_{m}\left(k_{1} a\right) J_{m}^{\prime}\left(k_{2} a\right)-k_{1} J_{m}^{\prime}\left(k_{1} a\right) J_{m}\left(k_{2} a\right)} & (p=q, \\
H_{m-n}^{(2)}\left(k_{1} r_{p q}\right) e^{-j(m-n) \phi_{p q}} & (p \neq n)
\end{array}\right.
\end{aligned}
$$

\section{B. Modal Analysis}

Consider a post-wall waveguide with infinite length, dividing the waveguide into equal unit cells (as shown in Fig. 2). The expansion coefficients of all cells are equal, except for a linear progressive phase constant $e^{j \nu k_{p} d_{x}}$ for the $\nu$-th cell. We construct a matrix for the infinite system, composed of the contributions of all cells with their phase differences taken into account. For specific values of the variable $k_{p}$, this system matrix will show singularities, because one of its eigenvalues approaches zero. The corresponding value of $k_{p}$ is the propagation constant of a propagating mode. 


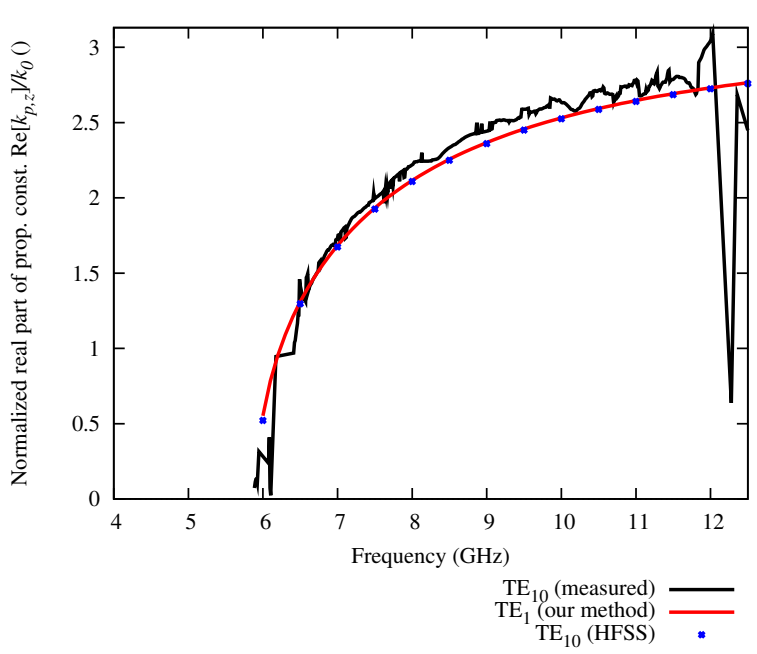

(a) real part

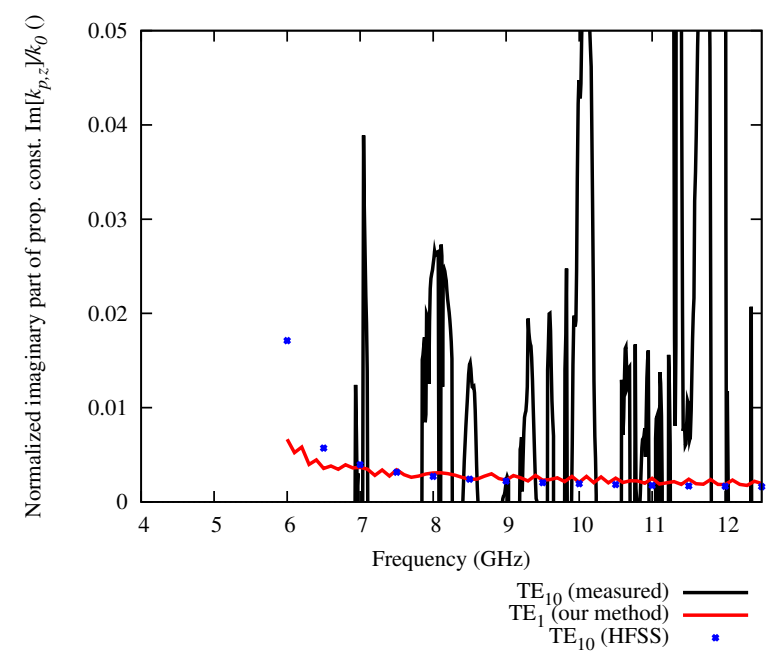

(b) imaginary part

Fig. 3. Normalized propagation constant as a function of frequency (metallic posts).

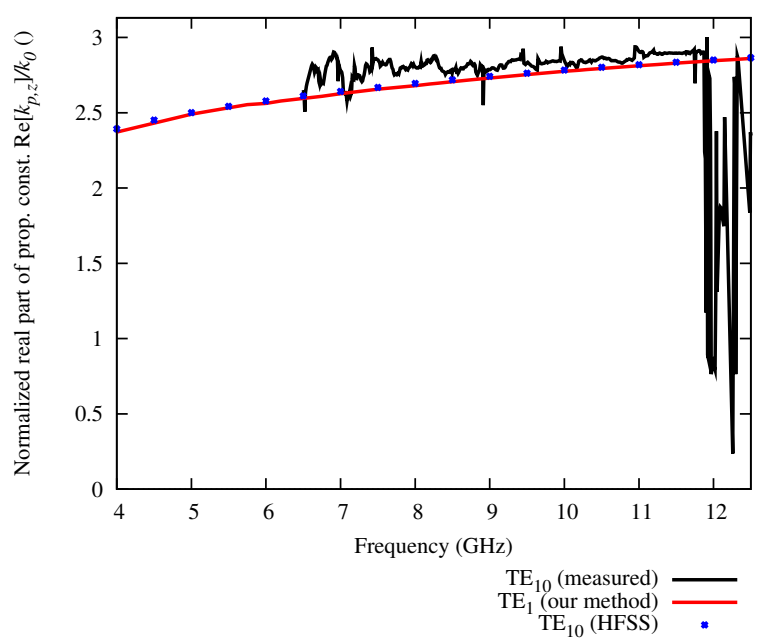

(a) real part

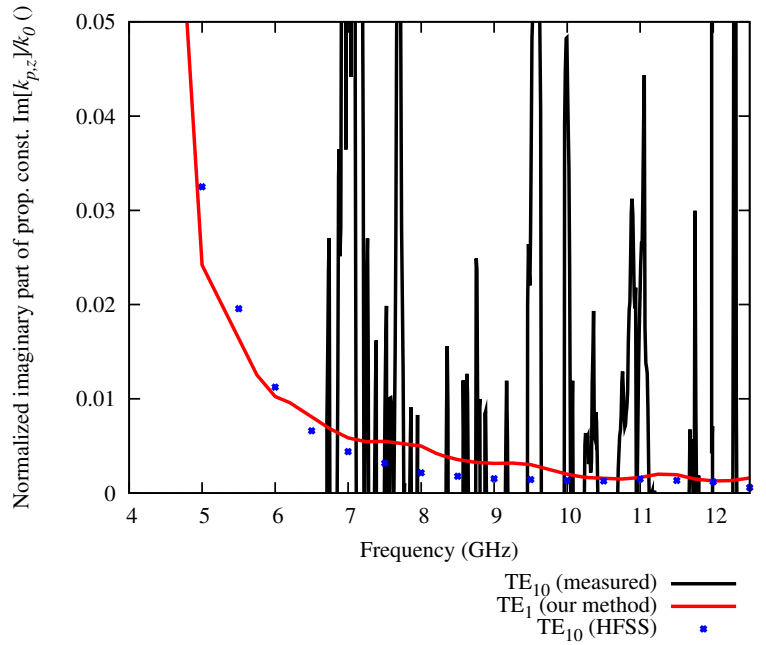

(b) imaginary part

Fig. 4. Normalized propagation constant as a function of frequency (dielectric posts).

\section{NUMERICAL RESULTS}

Two different post-wall waveguide geometries have been studied; both numerical simulations as well as manufactured test samples are investigated. One geometry is based on metallic posts and the other on dielectric posts. Their parameters are given in Table $\mathrm{I}, h_{g}$ is the height of the waveguide, $f_{\text {stop }}$ is the start frequency of the first stop band due to the periodicity, $M$ is the number of expansion terms in the sum of equation 1 and $L$ is the number of cylinders per side wall in a unit cell.

\section{A. Dispersion Characteristics}

We use the Newton-Raphson method to numerically find the modal propagation constants $k_{p}$, solving the characteristic equation for zero eigenvalue

$$
\operatorname{det}\left[\mathbf{C}^{\infty}\left(k_{p}\right)\right]=0 .
$$

TABLE I

GEOMETRY SPECIFICATIONS FOR THE ANALYZED AND MANUFACTURED POST-WALL WAVEGUIDES.

\begin{tabular}{|ll|l|l|}
\hline \multicolumn{2}{|c|}{ parameter } & metal cyl. & diel. cyl. \\
\hline$\epsilon_{r}, 1$ & () & 9.80 & 9.80 \\
$\epsilon_{r, 2}$ & () & - & 1.00 \\
$a$ & $(\mathrm{~mm})$ & 0.65 & 1.50 \\
$d_{x}$ & $(\mathrm{~mm})$ & 2.57 & 3.30 \\
$d_{y}$ & $(\mathrm{~mm})$ & - & 4.09 \\
$w_{g}$ & $(\mathrm{~mm})$ & 8.91 & 8.91 \\
$h_{g}$ & $(\mathrm{~mm})$ & 3.81 & 3.81 \\
$f_{\text {stop }}(\mathrm{GHz})$ & 18.63 & 14.51 \\
$M$ & () & 3 & 3 \\
$L$ & () & 1 & 3 \\
\hline \multicolumn{2}{|l}{} \\
\hline
\end{tabular}

In Fig. 3(a) the real part and in Fig. 3(b) the imaginary part of $k_{p}$ for the dominant $\mathrm{TE}_{10}$ mode normalized to the propagation constant of the background medium $k_{1}$ are plotted 
(red curves) for a post-wall waveguide with metal posts; in Fig. 4 these parameters are plotted for a post-wall waveguide with dielectric posts. The geometric specifications for these waveguides are shown in Table I. The post-wall waveguide with metallic posts, shows clearly a modal behavior that is similar to a normal rectangular waveguide with solid walls. In the case of the dielectric post-wall waveguide, the dominant mode is less dispersive and does not show a distinct cut off behavior, as in the case of the metallic posts.

\section{Measurements}

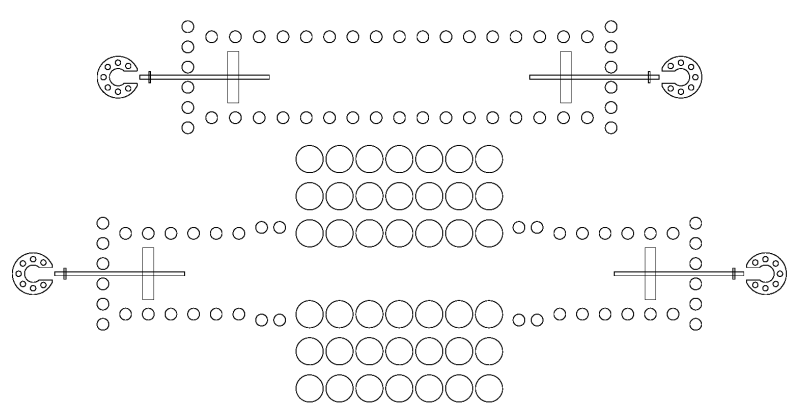

Fig. 5. Layout of segments of metallic and dielectric post-wall waveguide transmission line.

Our strategy is, as in the case of the HFSS simulations, based on the extraction of the propagation constant from the scattering parameters using multiline calibration [23]. Fig. 5 shows the layout and the measured PCB (Rogers TMM10i) with all the post-wall waveguide transmission lines. The postwall waveguides are fed through a slot-coupled microstrip line. The scattering parameters are measured using an Agilent NS230A PNA.

\section{Discussion}

Numerical results for the real part of the propagation constant, presented in Fig. 3 and 4, show excellent agreement. The measurements of $\operatorname{Re}\left(k_{p, z}\right)$ show reasonable agreement, until $f \approx 12 \mathrm{GHz}$, which is the cut off frequency of the $\mathrm{TE}_{20}$ mode in the metallic post-wall waveguide.

In the case of metallic posts, the numerical results for $\operatorname{Im}\left(k_{p, z}\right)$ show good agreement, but in the case of dielectric posts, the results from HFSS are lower than predicted by our own method. Measurements of the imaginary part of the propagation constant exhibit noise. This is the result of differences in attenuation loss between the incremental line lengths being often smaller than the measurement uncertainty.

\section{ACKNOWLEDGMENT}

We thank the Dutch Ministry of Economic Affairs, which supported this work within the scope of the WiComm project.

\section{REFERENCES}

[1] J. Hirokawa and M. Ando, "Single-layer feed waveguide consisting of posts for plane TEM wave excitation in parallel plates," IEEE Trans. Antennas Propagat., vol. 46, no. 5, pp. 625-630, May 1998.
[2] Y. Cassivi, L. Perregrini, P. Arcioni, M. Bressan, K. Wu, and G. Conciauro, "Dispersion characteristics of substrate integrated rectangular waveguide," IEEE Microw. Wireless Comp. Lett., vol. 12, no. 9, pp. 333-335, Sep. 2002.

[3] F. Xu, Y. Zhang, W. Hung, K. Wu, and T. Cui, "Finite-difference frequency-domain algorithm for modelling guided-wave properties of substrate integrated waveguide," IEEE Trans. Microwave Theory Techn. vol. 51, no. 11, pp. 2221-2227, Nov. 2003.

[4] $\mathrm{F}$. $\mathrm{Xu}$ and $\mathrm{K}$. Wu, "Numerical multimode calibration technique for extraction of complex propagation constants of substrate integrated waveguide," in IEEE MTT-S Int. Microwave Symp. Dig., Fort Worth, TX, Jun. 2004, pp. 1229-1232.

[5] — "Guided-wave and leakage characteristics of substrate integrated waveguide," IEEE Trans. Microwave Theory Techn., vol. 53, no. 1, pp. 66-73, Jan. 2005

[6] J. Hirokawa and M. Ando, "Efficiency of 76-GHz post-wall waveguidefed parallel-plate slot arrays," IEEE Trans. Antennas Propagat., vol. 48, no. 11 , pp. 1742-1745, Nov. 2000

[7] J. Simpson, A. Taflove, J. Mix, and H. Heck, "Substrate integrated waveguides optimized for ultrahigh-speed digital interconnects," IEEE Trans. Microwave Theory Techn., vol. 54, no. 5, pp. 1983-1990, May 2006.

[8] C. Tseng and T. Chu, "Measurement of frequency-dependent equivalent width of substrate integrated waveguide," IEEE Trans. Microwave Theory Techn., vol. 54, no. 4, pp. 1431-1437, April 2006.

[9] D. Deslandes and K. Wu, "Design consideration and performance analysis of substrate integrated waveguide components," in Proc. 32th Eur. Microw. Conf., vol. 2, Milan, Italy, Sep. 2002, pp. 881-884.

[10] H. Uchimura, T. Takenoshita, and M. Fujii, "Development of a "Laminated Waveguide"," IEEE Trans. Microwave Theory Techn., vol. 46 no. 12, pp. 2438-2443, December 1998.

[11] H. Benisty, "Modal analysis of optical guides with two-dimensional photonic band-gap boundaries," J. Appl. Phys., vol. 79, no. 10, pp. 74837492, May 1996

[12] A. Adibi, Y. Xu, R. Lee, A. Yariv, and A. Scherer, "Properties of the slab modes in photonic crystal optical waveguides," J. Lightwave Technol., vol. 18 , no. 11 , pp. 1554-1564, November 2000

[13] D. Pissoort, E. Michielsen, F. Olyslager, and D. D. Zutter, "Fast analysis of 2-d electromagnetic crystal devices using a periodic green function approach," J. Lightwave Technol., vol. 23, no. 7, pp. 2294-2308, July 2005.

[14] R. Hwang and C. Hsiao, "Frequency-selective transmission by a leaky parallel-plate-like waveguide," IEEE Trans. Antennas Propagat., vol. 54, no. 1, pp. 121-129, January 2006.

[15] I. El-Kady, M. Sigalas, R. Biswas, and K. Ho, "Dielectric waveguides in two-dimensional photonic bandgap materials," J. Lightwave Technol., vol. 17, no. 11, pp. 2042-2049, November 1999.

[16] H. Jia and K. Yasumoto, "Modal analysis of two-dimensional photoniccrystal waveguides formed by rectangular cylinders using an improved fourier series method," IEEE Trans. Microwave Theory Techn., vol. 54, no. 2, pp. 564-571, Feb. 2006

[17] A. Patrovsky and K. Wu, "Substrate integrated image guide (SIIG)a low-loss waveguide for millimetre-wave applications," in Proc. 35th Eur. Microw. Conf., Paris, France, October 2005, pp. 897-900.

[18] — "Substrate integrated image guide (SIIG) — a planar dielectric waveguide technology for millimeter-wave applications," IEEE Trans. Microwave Theory Techn., vol. 54, no. 6, pp. 2872-2879, June 2006.

[19] _ - "Substrate integrated image guide array antenna for the upper millimeter-wave spectrum," IEEE Trans. Antennas Propagat., vol. 55 no. 11, pp. 2994-3001, November 2007.

[20] T. Coenen, A. Mazzinghi, D. Bekers, A. Neto, J. Tauritz, A. Freni, and G. Gerini, "Design and analysis of EBG based integrated waveguide structures for microwave and MM-wave feed networks," in Proc. IEEE AP-S Int. Symp. Dig., Albuquerque, NM, July 2006, p. 654.

[21] T. Coenen, D. Bekers, J. Tauritz, and F. van Vliet, "Design of post-wall waveguides with metal and dielectric posts," in Proc. 37th Eur. Microw. Conf., Munich, Germany, Oct. 2007, pp. 20-23.

[22] A. Elsherbeni and A. Kishk, "Modeling of cylindrical objects by circular dielectric and conducting cylinders," IEEE Trans. Antennas Propagat., vol. 40, no. 1, pp. 96-99, January 1992.

[23] R. Marks, "A multiline method of network analyzer calibration," IEEE Trans. Microwave Theory Techn., vol. 39, no. 7, pp. 1205-1215, Jul. 1991. 